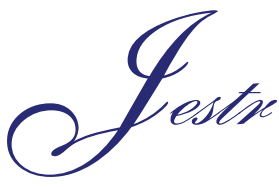

Review Article

\title{
Energy Efficiency in Underwater Sensor Networks: a Research Review
}

\author{
K. Ovaliadis*, N. Savage and V. Kanakaris \\ Department of Electronic and Computer Engineering, University of Portsmouth, Portsmouth, UK.
}

Received 11 March 2010; Revised 30 April 2010; Accepted 20 June 2010

\begin{abstract}
In an energy-constrained underwater system environment it is very important to find ways to improve the life expectancy of the sensors. Compared to the sensors of a terrestrial Ad Hoc Wireless Sensor Network (WSN), underwater sensors cannot use solar energy to recharge the batteries, and it is difficult to replace the batteries in the sensors. This paper reviews the research progress made to date in the area of energy consumption in underwater sensor networks (UWSN) and suggests further research that needs to be carried out in order to increase the energy efficiency of the UWSN system.
\end{abstract}

Keywords: Underwater acoustic sensor networks (UWSNs), Acoustic communications, Energy efficiency, Lithium-ion batteries, Acoustic modems, Underwater networking, Network topology, Medium access control (MAC), Routing protocols, Clustering.

\section{Introduction}

The interest in Ad Hoc Wireless Sensor Networks (WSNs) have been growing rapidly in the past few years and lot of research has been done on several areas, such as communications, power conservation, routing algorithms and protocols. Much of the research has focused on terrestrial Ad Hoc WSN with little attention given to Ad Hoc UWSN. Acoustic communications are the typical physical layer technology in underwater networks, providing robust underwater communications for both military and civilian applications. Some of these applications are: environmental monitoring, underwater exploration, disaster prevention, assisted navigation, tactical surveillance, and mine detection [1], [2], [3].

An underwater network is typically made up of many autonomous and individual sensor nodes that perform data collection operations as well as store and forwarding operations to route the data that has been collected to a central node. The main challenges of deploying such a network are the cost, the computational power, the memory, the communication range and most of all the limited battery resources of each individual sensor node. As the life time of any individual sensor in the UWSN is limited, the number of sensor nodes that stop working due to the power loss increases with a lengthened deployment time, therefore the coverage area of WSN will shrink. It is obvious that the issue of limited battery resources is particularly important and it is a challenge for researchers to obtain long operating time without sacrificing system performance. Therefore new, energy efficient protocols must be developed for all of the UWSN nodes' functions.

${ }^{*}$ E-mail address: kyriakos.ovaliadis@port.ac.uk

ISSN: 1791-2377 (C) 2010 Kavala Institute of Technology. All rights reserved.

\section{Review of similar research studies}

\subsection{Battery Technology}

To increase network lifetime, energy must be saved in every hardware and software solution composing the network architecture. One way to resolve the battery problem is for the UWSN sensor to generate energy by itself. This can be achieved by using chemistry or mechanical methods such as current movement. On the other hand if the type of battery is chosen, the Li-ion systems are the most promising technology for the underwater sensors mainly due to their higher energy and power densities compared to other technologies such as Nickel Cadmium and Nickel Metal Hydride. The main features of lithium ion technology are the low life cycle cost (long cycle life, no memory effect, no maintenance), the low discretion rate (no thermal or magnetic signature), the design flexibility (battery systems are independent, secured and communicant) and the readable battery status (with electronics and easy state of charge evaluation) [4]. Aside from analyzing the physical design characteristics of Li-ion cells and batteries, Yardney Technical Products, Inc. [5] have performed extensive research and testing on various chemistries suitable for underwater environment. Figure 1 compares Li-ion technology to other battery technologies. The "HP" bubble relates to the highest power systems, the "Typical Military" bubble corresponds to the general performance window of fielded military systems and the "HE" bubble corresponds to system where discharge rates are low and the temperature is no colder than $-20^{\circ} \mathrm{C}$.

The superiority of the Lithium ion technology was also apparent when used in an experiment instead of alkaline technology. I. Vasilescu et al. [6] built 20 underwater sensor nodes called Aquaflecks for their experiment. Each sensor node is powered by 


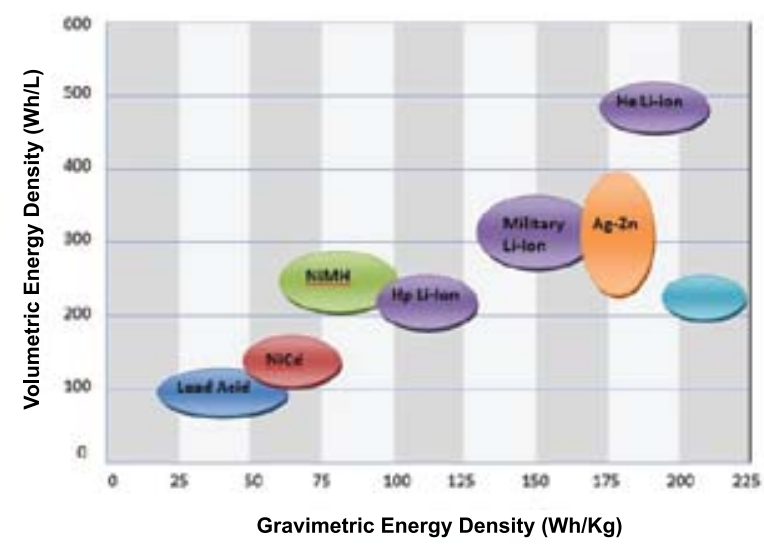

Figure 1. Battery Technology Energy Densities

three alkaline $\mathrm{C}$ cells. Three $\mathrm{C}$ cells can provide $27 \mathrm{Wh}$ and four days of continuous operation with all sensors and communication hardware fully powered. I. Vasilescu et al. [7] has also built a second generation underwater sensor network called AquaNodes. This time each sensor node is powered by seven 2 amp/hour Lithium ion batteries. In comparison to the previous experiment, when all the components of the node run at full power the battery provides 2 weeks of continuous operation.

\subsection{Modem technology}

Similar to the terrestrial, in the underwater sensor network the modem is responsible for implementing the physical layer of the network stack as shown in Figure 2. The modem is responsible for the data transmission and reception across the network, while the higher network layers are responsible for MAC protocols (link layer), routing protocols (network layer), transport protocols (transport layer), and data processing (application layer).

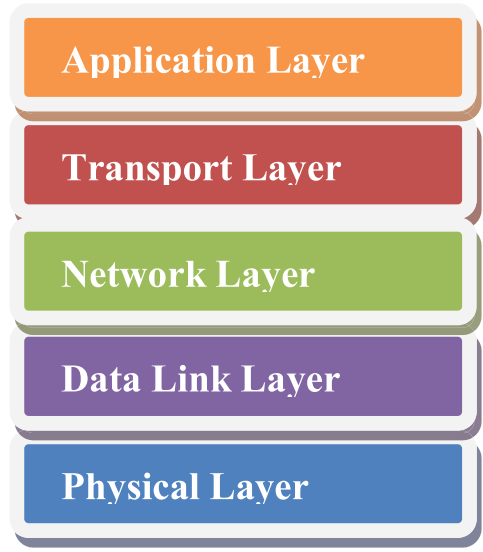

Figure 2. Typical underwater network stack

Nowadays few underwater sensor networks exist because commercially available underwater acoustic modems are too costly and energy inefficient to be practical for this applications. The commercially available acoustic modems provide data rates ranging from $100 \mathrm{bps}$ to about $40 \mathrm{Kbps}$, and they have an operating range of up to a few $\mathrm{km}$ and an operating depth in the range of thousands of meters. The cost of a single commercial underwater acoustic modem is at least a few thousand US dollars. Therefore, when designing an acoustic modem for sensor networks, the de- signer must optimize for low cost and low energy consumption at every level, from the analog electronics to the signal processing scheme. The design choices that must be considered include the choice of DSP scheme, the choice of underwater transducer and corresponding analog electronics, the choice of interfaces to sensors, and the choice of hardware platform for the implementation.

A major research in underwater acoustic modems has already been made using a variety of different hardware platforms, including digital signal processors [8] and microcontrollers [9].

Freitag, et al. [8], have developed a compact and low-power acoustic modem, called Micro-modem. The Micro-modem is based on a Texas Instruments fixed-point DSP, consuming about $180 \mathrm{~mW}$ when fully active. The transmission power is fixed and built into the hardware for each application. A unique feature of the Micromodem is that it has two operating modes: 1) low-power, low-rate and non-coherent (frequency hopping-FSK) and 2) high-power, high-rate and coherent (phase-shift keying). The Micro-modem also incorporates basic acoustic navigation functionality.

Jack Wills et al. [9], have developed a new inexpensive low power acoustic modem targeted particularly at supporting shortrange acoustic communication for dense underwater networks. The main power-saving innovation is to use a dedicated, very low-power, all-analog wakeup tone receiver to trigger the more expensive data receiver. When there is no communication activity, nodes can turn off most components, and only leave the wakeup receiver on.

It can be concluded that Micro-modem is much more advanced for physical layer research. However, the modem of $\mathrm{J}$. Willis et al. consumes less power and has a much simpler design; although this modem is designed only for short range communication $(50-500 \mathrm{~m})$.

\subsection{Network Topology}

The network topology is in general a crucial factor in determining the energy consumption, the capacity and the reliability of a network. The main objective of an energy efficient topology scheme is to increase network lifetime by reducing the overall or individual energy consumption of nodes. This can be achieved by using an optimal topology deployment scheme (i.e. minimum number of sensors to be deployed), or an optimal topology management scheme (i.e. form a cluster), or both schemes combined. Hence, the network topology should be carefully engineered and post-deployment topology optimization should be performed, when possible.

A two group categorization can be made according to a sensor's mobility. 1) Static UWSNs, in which sensors are anchored after deployment; 2) Mobile UWSNs with free-floating sensors.

\subsubsection{Static UWSN}

The main characteristic of static architecture is that the sensors would be relatively static after deployment, meaning negligible movement. The network could be anchored into two-dimensional (2D) or three-dimensional (3D) space [10].

\section{A. Two-dimensional space}

In the $2 \mathrm{D}$ case, the topology could be grid, cluster, tree, or linerelay deployment. Figure 3 shows an underwater sensor network organized in a cluster-based scheme. Each sensor is interconnected 
to one or more head sensors (gateways) by using wireless acoustic links. The head sensors are network devices in charge of relaying data from the ocean bottom network to a surface station [10].

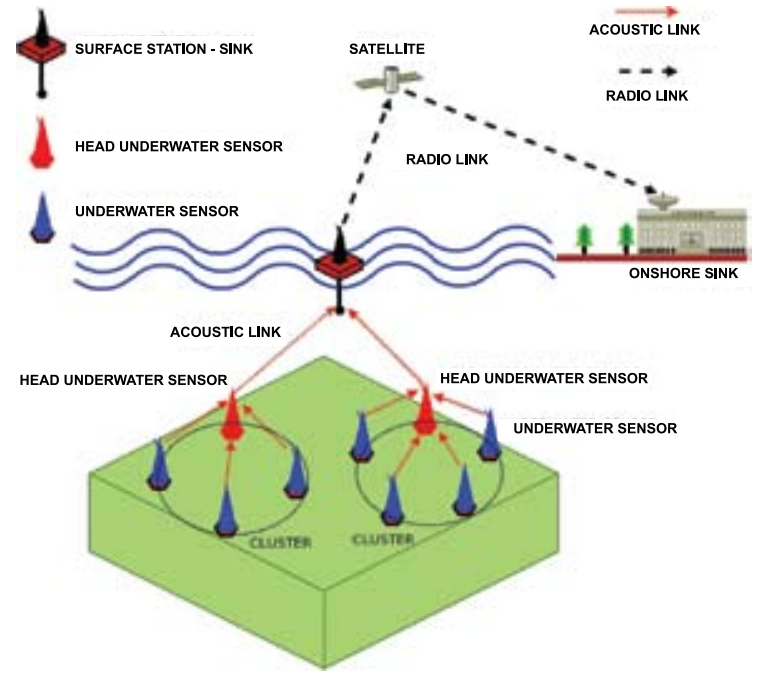

Figure 3. Two-dimensional (2D)

To increase the robustness and energy efficiency, a two dimensional multi-tier topology was proposed by W. K. G. Seah and H. X. Tan [11]. The topology consists of sensor clusters where each cluster has one or more local aggregation points. These aggregation points, called virtual sinks, form a mesh network. Sensors transmit their data through multi-hop to local sink via virtual sink within their own cluster. For a given multi-tier topology it has been shown that the multipath approach always incurs lower latency and is more reliable and energy efficient when the channel becomes harsh.

At this point it can be said that clustering is one good method that can lead to a more energy efficient and reliable underwater network. It is a method proposed by many researchers that provides a convenient framework for resource management. It can support many important network features, such as channel access for cluster members, power control and routing. On the other hand it has not been widely yet on mobile underwater sensors.

\section{B. Three-dimensional space}

In the 3D case (figure 4), sensors could be moored to anchors on the ocean floor or to surface floats with fix depth. Threedimensional underwater networks are used to detect and observe phenomena that cannot be adequately observed by means of ocean bottom uw-sensor nodes, i.e., to perform cooperative sampling of the $3 \mathrm{D}$ ocean environment [10].

For $3 \mathrm{D}$ cases a very innovated approach has been proposed by I. F. Akyildiz et al. [12]. Winch based sensors are anchored to the ocean bottom, equipped with a floating buoy that can be inflated by a pump. The buoy pulls the sensor towards the ocean surface. The depth of the sensor can then be regulated by adjusting the length of the wire that connects the sensor to the anchor. Three deployment strategies have been proposed: the 3D random, the bottom random and the bottom grid. In the first two, the sensors are randomly deployed on the bottom, where they are anchored however in the bottom grid strategy sensors needs to be assisted by one or multiple AUVs, which deploy the underwater sensors

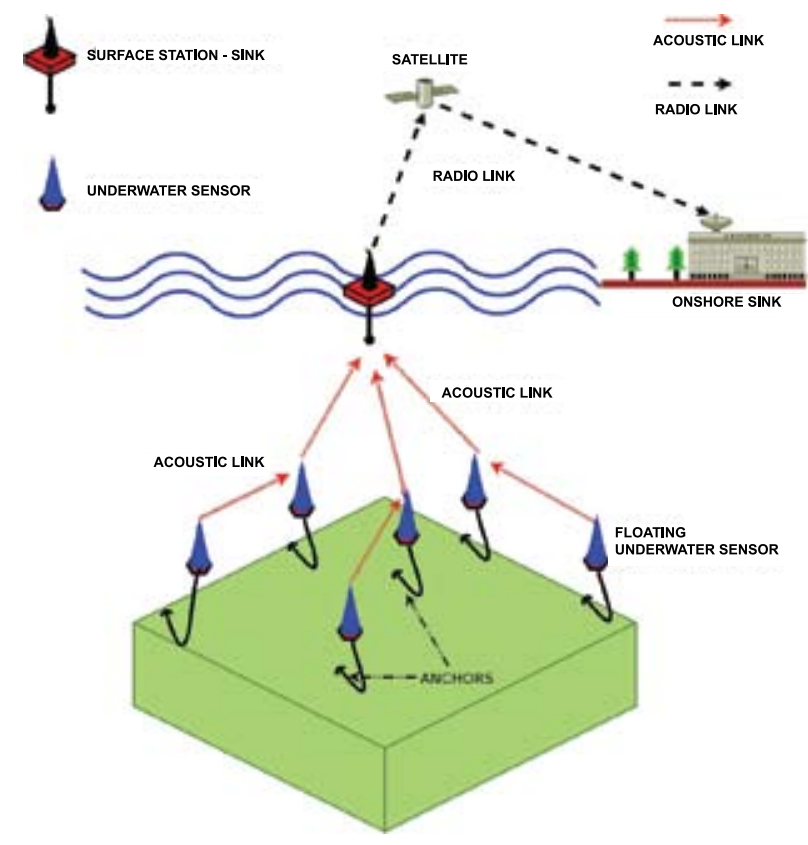

Figure 4. Three-dimensional (3D)

to predefined target locations to obtain a grid deployment on the bottom of the ocean. Simulation results show that the coverage ratio obtained with the bottom-grid strategy is greater than the coverage ratio obtained with the bottom-random strategy and the 3D-random strategy. Moreover, given a target coverage ratio, the minimum number of sensors needed to achieve the desired coverage ratio decreases with the complexity of the deployment strategy. Decreasing the number of the sensors could be lead to more energy efficient network.

\subsubsection{Mobile UWSN}

In comparison to the static, a mobile UWSN is a self-organizing network. Underwater sensor nodes may be redistributed and moved by the aqueous processes of advection and dispersion. After transport by the currents and dispersion, the sensors must reorganize as a network in order to maintain communication [13].

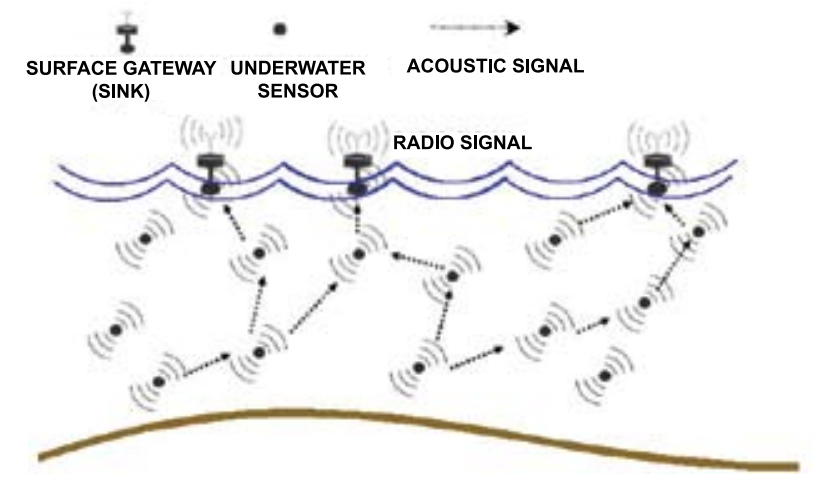

Figure 5. A Mobile Underwater Sensor Network

In [13], the authors introduce the following two classifications for mobile underwater sensor networks:

- Mobile UWSNs for long-term non-time-critical aquatic monitoring: These include networks of local underwater sensors that collect data and relay them to intermediate underwater sensors; these nodes forward the packets to the surface nodes, 
which transmit data, for example via radio, to the on-shore command center. Typical applications may be oceanography, marine biology, deep-sea archaeology, seismic predictions, pollution detection and oil/gas field monitoring.

- Mobile UWSNs for short-term time-critical aquatic exploration: These include networks of underwater sensors that collect data and forward them to the surface control center via multi-hop acoustic routes. Typical applications may be underwater natural resource discovery, hurricane disaster recovery, anti-submarine military mission and loss treasure discovery.

\subsection{MAC protocols}

MAC layer has the objectives of managing and controlling communication channels, which are shared by many nodes to avoid collisions and maintain reliable transmission condition. Due to the dense deployment of sensors in UWSNs, it is necessary to design an efficient medium access control (MAC) protocol to coordinate the communication among sensors. In short, a viable MAC solution for UWSNs should take long propagation delay, low available bandwidth, energy efficiency (for long-term applications) and node mobility (for mobile UWSNs) into account. So far, various approaches have been explored.

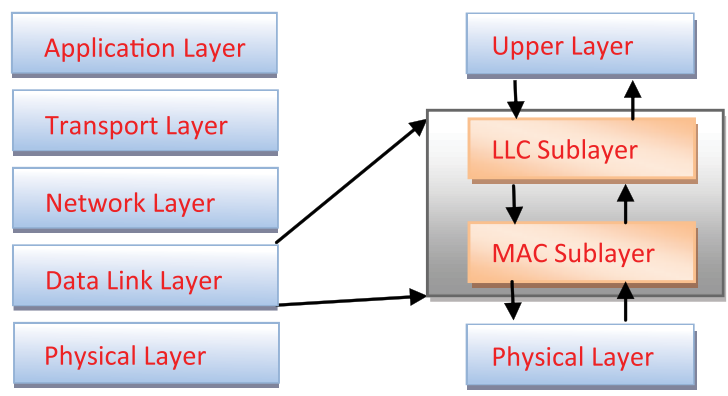

Figure 6. Data link layer architecture in OSI reference model

MAC layer protocols can be classified in two main categories: contention based and schedule based protocols. Schedule based protocols include TDMA, FDMA and CDMA, where communication channels are separated in time, frequency or code domains. This kind of protocols are class of deterministic MAC layer protocol in which access to the channel is based on a schedule and it is limited to one user at a time. Contention-based protocols include random access methods and collision avoidance methods. In a random access protocol, the sender sends packets without coordination. Thus packet avoidance is totally probabilistic. While in a collision avoidance protocol, the sender and receiver capture the medium through control packet exchange before data transmission [14].

Among these protocols, existing MAC solutions are mainly focused on CSMA or CDMA. Frequency Division Multiple Access (FDMA) is not suitable for UW-ASN due to the narrow bandwidth in underwater channels and the vulnerability of limited band systems to fading and multipath. Moreover, Time Division Multiple Access (TDMA) shows limited bandwidth efficiency because of the long time guards required in the underwater channel. Furthermore, the variable delay makes it very challenging to realize a precise synchronization, with a common timing reference [13], [14].

\subsubsection{Contention based MAC protocols}

A medium access control (MAC) protocol suitable for an underwater acoustic network is proposed and analyzed by M. Molins and M. Stojanovic [15]. Slotted FAMA is based on a channel access discipline called floor acquisition multiple access (FAMA). It combines both carrier sensing (CS) and a dialogue between the source and receiver prior to data transmission. During the initial dialogue, control packets are exchanged between the source node and the intended destination node to avoid multiple transmissions at the same time. Although time slotting eliminates the asynchronous nature of the protocol and the need for excessively long control packets, thus providing savings in energy, guard times should be inserted in the slot duration to account for any system clock drift. In addition, due to the high propagation delay of underwater acoustic channels, the handshaking mechanism may lead to low system throughput, and the carrier sensing may sense the channel idle while a transmission is still going on.

A distributed energy-efficient MAC protocol tailored for the underwater environment was proposed by V. Rodoplu and M. K. Park [16], whose objective is to save energy based on sleep periods with low duty cycles. The proposed solution is strictly tied to the assumption that nodes follow sleep periods, and is aimed at efficiently organizing the sleep schedules. This protocol tries to minimize the energy consumption and does not consider bandwidth utilization or access delay as objectives.

\subsubsection{Schedule based MAC protocols}

UW-MAC, a distributed MAC protocol for underwater acoustic sensor networks, was proposed by D. Pompili et al. [17]. It is transmitter based CDMA schemes that incorporates a closed-loop distributed algorithm to set the optimal transmit power and code length. It is proven that UW-MAC manages to simultaneously achieve high network throughput, limited channel access delay, and low energy consumption. Nevertheless according to the authors there are some open research issues: it is necessary to design access codes with high auto-correlation and low cross-correlation properties to achieve minimum interference among users and research must be done on optimal data packet length, to maximize the channel utilization efficiency.

A. A. Sayed et al [18] propose an energy-efficient MAC protocol for short range, acoustic sensor networks called "Tone Lohi". TLohi provides an energy conserving, throughput efficient, fair, and stable medium access for acoustic networks. The energy is conserved in two ways: firstly, using data reservations to ensure no data packets collide. Secondly, employing wake-up tone hardware that resolves reservation contention with extremely low energy cost. Stability and throughput efficiency was achieved by employing a mechanism that provides collision detection and contender count allowing the use of an intelligent back off mechanism that reduces the overall time for fairly reserving data. Three versions of TLohi representing different design choices were proposed and tested. Simulation results show that ST-Lohi (Synchronized T-Lohi) is the most energy efficient protocol, within 3\% of optimal energy. aUT-Lohi (Aggressive Unsynchronized T-Lohi) achieves the highest throughput ( $\sim 50 \%$ channel utilization). cUT-Lohi (Conservative Unsynchronized T-Lohi) provides the most robust packet delivery with almost no packet loss. All three versions exhibit efficient channel utilization, stable throughput, and excellent energy efficiency. 


\subsection{Routing Protocols}

One of the main design goals of UWSNs is to carry out data communication while trying to prolong the lifetime of the network. Therefore an important research issue is the design of robust, scalable and energy-efficient routing protocols. The existing routing protocols are usually divided into three categories, namely proactive (e.g., DSDV [19]), reactive (e.g., AODV [20]), and geographical routing protocols (e.g., GFG [21]). The first two categories are unsuitable for UWS networks because they apply a continuous exchange of overhead messages or employ a route discovery process based on the flooding technique; these mechanisms are inefficient tools in large scale underwater networking because they consume excessive energy and bandwidth resources. However the third category could be applied to underwater environments if it is investigated how sensor nodes can obtain accurate localization information without much power consumption; the extended Global Positioning System (GPS) is not helpful to achieve this purpose because it uses radar waves in the $1.5 \mathrm{GHz}$ band and those waves do not propagate in sea water [22].

Some recent papers propose routing protocols specifically tailored for underwater acoustic networks. E. Sozer et al. [23], propose a simple design example of a shallow water network, where routes are established by a central manager based on neighborhood information gathered from all nodes by means of poll packets. The master node collects the neighbor tables from all nodes in the network and uses this information to establish a routing tree and decide on the primary and secondary routes to each destination. The master node is responsible for sending the primary routes to all nodes. However this routing protocol assumes that the nodes are static and cannot be properly applied to large-scale mobile UWSNs because routes will break frequently due to mobility.

P. Xie et al. [24] propose a robust, scalable and energy efficient routing protocol, called vector-based forwarding (VBF), to address the routing problem in UWSNs. VBF is essentially an integration of localization and routing in that the localization and routing are performed at the same time. In essence, VBF is a geographic routing approach, where no state information is required on the sensor nodes and only a small fraction of the nodes are involved in routing. In VBF, each packet carries the positions of the sender, the destination and the forwarder. The forwarding path is specified by the so-called routing vector, (a vector that connects source and destination). Upon receiving a packet, a node computes its position relative to the forwarder by measuring its distance to the forwarder and the angle of arrival of the signal. Recursively, all the nodes receiving the packet compute their positions. If a node determines that it is close enough to the routing vector, it includes its own position in the packet and forwards it; otherwise, it discards the packet. In this way, all packet forwarders form a "routing pipe". All the sensor nodes in the pipe are potential forwarders for the packet and those which are not close enough to the routing vector, which constitutes the axis of the pipe, do not forward the packet. Packets are thus forwarded along redundant and interleaved paths from source to destination, which makes the protocol robust against packet loss and node failure. The proposed algorithm, however, does not consider applications with different requirements.

Chenn-Jung Huang et al. [25], developed a novel cluster- ing algorithm that combines the ideas of energy-efficient clusterbased routing and application specific data aggregation to achieve good performance in terms of system lifetime, and applicationperceived quality. The proposed clustering technique organizes sensor nodes into direction sensitive clusters, with one node acting as the cluster head that fit the unique characteristic of up/ down transmission direction in UWSNs. The cluster head node receives information from all the cluster members and transmits information to the head nodes of other clusters along the way to the sink node. A series of experiments were conducted to compare the proposed clustering algorithm with a representative clustering algorithm, LEACH (Low-Energy Adaptive Clustering Hierarchy) [26], and a routing protocol for UWSNs, vector based forwarding (VBF) [24]. The experimental results showed that the proposed work achieves better performance than the two representative schemes including number of nodes alive over time, total amount of data received at the sink over time, and the total data received at the sink node for a given amount of energy.

A Distributed Underwater Clustering Scheme (DUCS) has been proposed by M. C. Domingo and R. Prior [27], [28]. DUCS is simple, energy aware and GPS-free routing protocol specifically designed for long-term non-time-critical aquatic monitoring applications in underwater environments. In DUCS the nodes organize themselves into local clusters and one node is selected as cluster head for each cluster. All non-cluster head nodes transmit their data to their cluster-head via a single hop, while the cluster-head node receives data from all cluster members, performs signal processing functions on the data and transmits the data to the sink using multihop routing. Nodes close to each other process very frequently correlated data because they monitor the same phenomena and with the aid of data aggregation techniques the effective non-redundant data can be extracted by the cluster-head and send to the sink.

After the clustering creation process is over, each clusterhead coordinate the data transmissions in its own cluster by setting up a TDMA schedule and transmitting this schedule by using CDMA to the cluster members. The combination of DUCS with TDMA/CDMA has as a result the reduction of the interference and the improvement of the communication quality. Furthermore DUCS minimizes the data loss between sensors when it compensates the high propagation delays of the underwater medium by using a continually adjusted timing advance combined with guard time values. Forty simulations were conducted to compare the proposed scheme with the representative clustering algorithm, LEACH [26]. The experimental results showed that DUCS achieves a very high packet delivery ratio while considerably reduces the network overhead and increases the throughput.

\section{Open Research Issues}

As already stated, the common problems among UWSN architectures are reliability, energy consumption and cost. Sensors used for the underwater environment are more expensive than those used for the terrestrial environment. This implies that UWSNs would be small-scale and sparse networks. The network also requires reliable and balanced energy consumption to prolong network lifetime and increase network capacity. Finally the network topology should be reconfigured to guarantee network connectivity and ef- 
fective communication when some nodes lose their ability.

Even more in the static deployment case, several questions must be answered. One of these questions is what is the minimum number of sensors required to optimize the cost, in association with the application demand? Another question is what kind of topology could fulfill the application demand if all of the sensing nodes are uniformly distributed? How to optimize coverage is one of the most important questions that must be answered; especially how to ensure coverage if nodes become unavailable. Also an energy balance routing protocol is needed in order to prolong the network lifetime.

In networks that consist of free-floating mobile sensors, the situation is more difficult. Due to the random motion of the sea currents, the nodes often move beyond the communication range of each other, thus the following major questions should be answered: how to achieve real-time monitoring, how many nodes should be deployed to keep network connectivity in monitoring region and how to make energy computation through the longtime task.

\section{Conclusion}

This review paper has summarized the on-going research in underwater sensor networks, including research challenges in the direction of energy consumption. It can be concluded that due to the economic concern and the complex underwater environment, an UWSN should have the capacity to adjust itself to the changing environment. This goal can be achieved if the topology and the protocol design can be able to self-adaptive when environment changes. However both topology and routing design should always take into consideration energy efficiency which is critical to an UWSN life and normal operation.

\section{References}

1. X. Yang, K. G. Ong, W. R. Dreschel, K. Zeng, C. S. Mungle and C. A Grimes, "Design of a wireless sensor network for long-term, in-situ monitoring of an aqueous environment", pp. 455-472, Sensors 2 (2002), vol. 11.

2. N. N. Soreide, C. E. Woody and S. M. Holt, "Overview of ocean based buoys and drifters: Present applications and future needs", in: $16^{\text {th }}$ International Conference on Interactive Information and Processing Systems (IIPS) for Meteorology, Oceanography, and Hydrology, Long Beach, CA, USA, January 2004.

3. L. Freitag, M. Grund, C. V. Alt, R. Stokey and T. Austin, "A Shallow Water Acoustic Network for Mine Countermeasures Operations with Autonomous Underwater Vehicles", In Underwater Defense Technology (UDT), 2005.

4. R. Gitzendanner, F. Pugliaa, C. Martina, D. Carmena, E. Jonesa, S. Eavesb, "High power and high energy lithium-ion batteries for under-water applications", Journal of Power Sources, vol. 136, pp. 416-418, October 2004.

5. Yardney Technical Products, Inc. (2007, July), "Design and performance of lithium ion cells for underwater energy storage and power delivery", online: http://www.yardney.com/Lithion

6. I. Vasilescu, M. Dunbabin, P. Corke, K. Kotay and D. Rus, "Data collection, storage, and retrieval with an underwater sensor network", in Proc. ACM Sensys 2005, San Diego, CA, USA, November 2005.

7. I. Vasilescu, C. Detweiler and D. Rus, "AquaNodes: An Underwater Sensor Network", in Proc. WUWNet 2007, Montreal, Canada, September 2007.

8. Lee Freitag, Matthew Grund, Sandipa Singh, James Partan, Peter Koski, and Keenan Balldf, "The whoi micro-modem: An acoustic communications and navigation system for multiple platforms", in Proc. IEEE/MTS OCEANS Conference, Washington DC, USA, September 2005.

9. J. Wills, W. Ye, and J. Heidemann. "Low Power Acoustic Modem for Dense Underwater Sensor Networks," in Proc. IEEE Workshop on Wireless Underwater Networks (WUWNet06), September 2006.

10. D. Pompili, T. Melodia and I. F. Akyildiz, "Deployment Analysis in Underwater Acoustic Wireless Sensor Networks," in Proc. of ACM International Workshop on Underwater Networks, Los Angeles, CA, September 2006.

11. W. K. G. Seah and H. X. Tan, "Multipath Virtual Sink Architecture for Underwater Sensor Networks", in Proc. of 2002. IEEE OCEANS Asia Pacific Conference, Singapore, May 2006.

12. I. F. Akyildiz, D. Pompili, and T. Melodia, "Underwater Acoustic Sensor Networks: Research Challenges", Journal of Ad Hoc Networks, vol. 3(3), pp. 257-279, Elsevier, Amsterdam, May 2005.

13. I. F. Akyildiz, D. Pompili and T. Melodia, "State of the Art in Protocol Research for Underwater Acoustic Sensor Networks," ACM Mobile Computing and Communication Review, vol. 11, No. 4, October 2007.

14. J. Heidemann, Y. Li, A. Syed, J. Wills, and W. Ye., "Underwater sensor networking: Research challenges and potential applications", in Proc. of the IEEE Wireless Communications and Networking Conference, Las Vegas, Nevad, USA, April 2006.

15. M. Molins and M. Stojanovic, "Slotted FAMA: a MAC Protocol for Underwater Acoustic Networks", in Proc. MTS/IEEE OCEANS, Conference and Exhibition for Ocean Engineering, Science and Technology, Boston, MA, September 2006.

16. V. Rodoplu and M. K. Park, "An Energy-Efficient MAC Protocol for Underwater Wireless Acoustic Networks", in Proc. MTS/IEEE OCEANS, September 2005.

17. D. Pompili, T. Melodia and I. F. Akyildiz, "A Distributed CDMA Medium Access Control for Underwater Acoustic Sensor Networks", in Proc. of Mediterranean Ad Hoc Networking Workshop (Med-Hoc-Net), pp. 63-70, Corfu, June 2007.

18. A. A. Syed, W. Ye and J. Heidemann, "T-Lohi: A New Class of MAC Protocols for Underwater Acoustic Sensor Networks", in Technical Report ISI-TR-638, USC/Information Sciences Institute, April 2007.

19. C. Perkins and P. Bhagwat, "Highly Dynamic Destination Sequenced Distance Vector Routing (DSDV) for Mobile Computers", in Proc. Of ACM SIGCOMM, London, UK, 1994.

20. C. Perkins, E. Belding-Royer and S. Das, "Ad Hoc On Demand Distance Vector (AODV) Routing", IETF RFC 3561.

21. P. Bose, P. Morin, I. Stojmenovic and J. Urrutia, "Routing with Guaranteed Delivery in Ad Hoc Wireless Networks", ACM Wireless Networks, 7(6):609-616, November 2001.

22. M. C. Domingo and R. Prior, "Energy analysis of routing protocols for underwater wireless sensor networks", Computer Communications, vol. 31, Issue 6, pp. 1227-1238, April 2008.

23. E. Sozer, M. Stojanovic, J. Proakis, Underwater acoustic networks, IEEE Journal of Oceanic Engineering 25 (1) (2000) 72-83.

24. P. Xie, J.-H. Cui, L. Li, "VBF: vector-based forwarding protocol for underwater sensor networks", UCONN CSE Technical Report: UbiNetTR05-03 (BECAT/CSE-TR-05-6), February 2005.

25. C. J. Huang, Y. J. Chen, I-Fan Chen, K. W. Hu, J. J. Liao, D. X. Yang, "A Clustering Head Selection Algorithm for Underwater Sensor Networks", in Proc. of FGCN '08, vol 1, pp. 21-24, December 2008.

26. W. Heinzelman, A. Chandrakasan, H. Balakrishnan, "An ApplicationSpecific Protocol Architecture for Wireless Microsensor Networks", IEEE Transactions on Wireless Communications 1 (4) (2002) 660-670.

27. M. C. Domingo, R. Prior, "A distributed clustering scheme for underwater wireless sensor networks", in Proc. of $18^{\text {th }}$ Annual IEEE International Symposium on Personal, Indoor and Mobile Radio Communications (PIMRC 2007), Athens, Greece, September 2007.

28. M. C. Domingo, R. Prior, Design and analysis of a gps-free routing protocol for underwater wireless sensor networks in deep water, in Proc. of UNWAT 2007, Valencia, Spain, October 2007. 